\title{
Inbalt.
}

Spite

Dritte Entmidelungaperiode oer griedifd: römif中en $\mathfrak{p h i l o j o p h i e ~ . ~ . ~ . ~ . ~ . ~} 1$

\section{Erfer $\mathscr{A}$ bidnitt.}

Die epifureifhe Eehre

Die fpäteren Epifureer

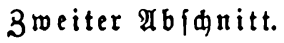

\section{Die Stoiler}

Beno's (Srundrinien bes froijden Segrgebäubes . $\quad$. $\quad$. 59

צusbau bes ftoifden Regrgebäubes . . . . $\quad . \quad 75$

Begriff und Blieberung ber Philojophie . $\quad . \quad$. 80

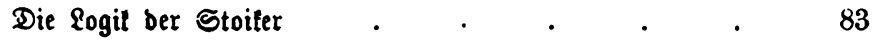

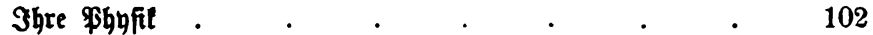

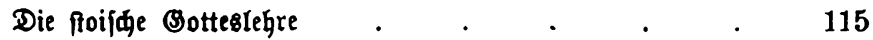

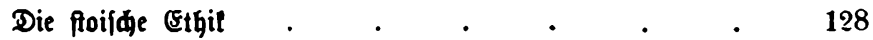

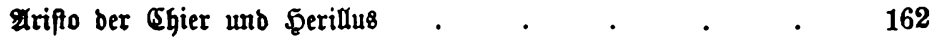

Bergleiđung bes froifhen \&egrgebäubes mit bem epitureifdjen uno

Dem platonifdsariftotelifden . . . . . . 165

\section{Dritter $\mathfrak{A} b\{\mathfrak{d} \mathfrak{n}$ itt.}

Die griedifde Slepfis

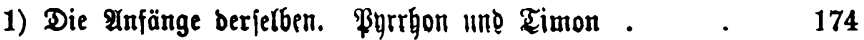

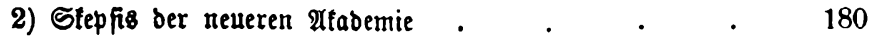

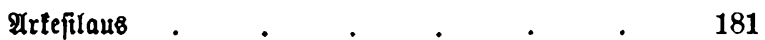

Rarneabes unb feine Sdule $\quad . \quad$. $\quad . \quad 184$

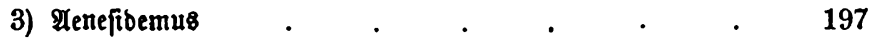

Igrippa und Menodotus . $\quad . \quad$. $\quad . \quad$. 208

Sertus ber Empirifer . . . . . . 209

Bergleidung ber Stepfis der Afabemiter und ber Pyrrgonier $\quad 230$ 
Bierter $\mathscr{4} b$ idnitt.

Seite.

Die Efleltif uno Synfretifit

1) Ulebergang ber Stoifer Dazu. Banätius unઠ Bofidonius

Uebergang ber ffeptijhen Rtabemie Jur Eflettit. Bhilo und Intiodus

Elleftil ber ßeripatetifer . $\quad . \quad$. $\quad . \quad$. $\quad 245$

Die griedjidge $\mathfrak{P h i l o l o p h i c ~ i n ~ R o m . ~} \mathfrak{R}$. Tullius Sicero 247

Die Sextier

2) \$eiterer Berlauf der Elleftil im erfen und jweiten $3 a b r$, Gundert. Die Stoiter biefer Sabrhunterte

\&. Innäus Senela $\quad$. $\quad$. $\quad . \quad$. $\quad 257$

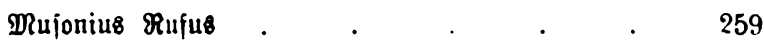

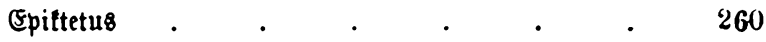

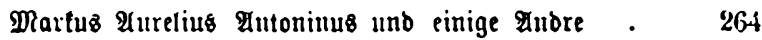

Die Thătigteit ber Beripatetiler biejer Jaḅrbunterte $\quad$. 267

Platonifer oer innfretififaen Rid)tung . . . . 268

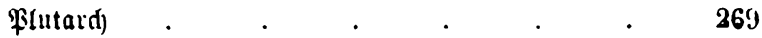

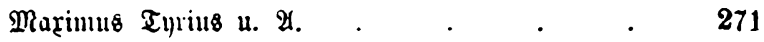

Silaudius Balpuus . . . . . . . 272

3) כyntretiftit unter Einnvirtıng Des Drients . . 273

Hilutard) u. e. $\mathscr{X}$. $\quad$. $\quad$. $\quad$. $\quad . \quad . \quad 273$

Einmirtung Des Drients auf bie griedildge Philofophic 276

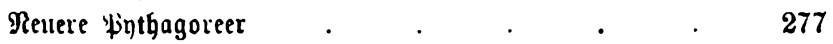

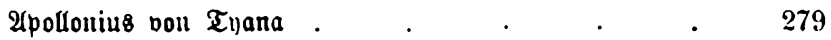

Die vom Srient ausgehende Ridtung. Whilo dir Sube 280

Die Emanationzleqre Der Bnofiter. Bafilites . . 293

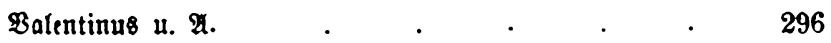

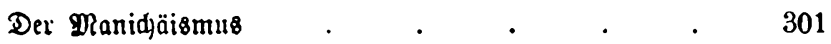

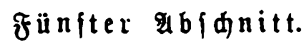

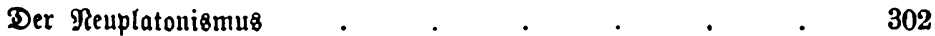

1) Unnuttelbare Borgänger beffelben, Numenius u. $\mathscr{x}$. . 303

llebergang 3 u Plotinus . $\quad . \quad$. $\quad . \quad$. 308

2) Plotimus; fein Seben und feine Sd)riften . 310 u. $320 \mathrm{ff}$.

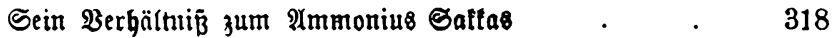

Blotinus Erfeuntniß̧lehre . $\quad . \quad$. $\quad$. 322

Eeine Segre von ben Principien; bas Innberingte . $\quad 330$

Der Beift . . . . . . . $\quad 340$

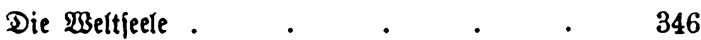


Seite.

Bon ber 2Belt ber Erifeinungen und bem Stoffe; vom

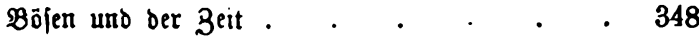

Die Welt und die Boriegung . . . . . . $\quad . \quad$. 352

Aftrologie und Dämonologie . $\quad . \quad$. $\quad . \quad$. 354

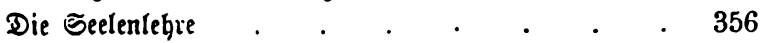

unferblidfeit ber Eccle und iqre Sdjiffale nad bem

Tode. . . . . . . . . 367

Einleitung in bic Ethit . $. \quad . \quad . \quad . \quad . \quad 370$

Blotins \&egre vom Sdjöten und von ber \&iebe .375

Die Dialettit als Mittel zur Erhebung bes Beiftes . 380

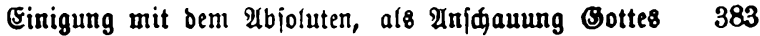

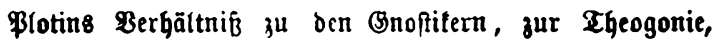
Pntbologie uno Dem Bolfsglauben, zum Sebet, zur Magie und Mantil, jur Stoa und Emanationslebre,

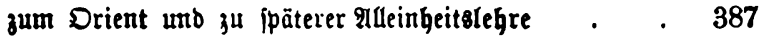

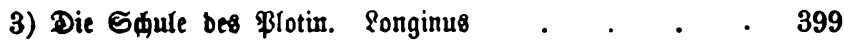

Imelius . . . . . . . . 400

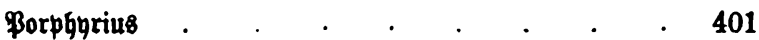

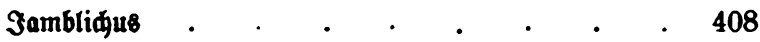

Qaifer Sulianus uno jeine Angänger . . . 413

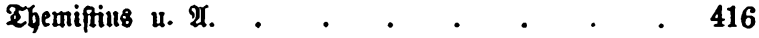

Die athenifde Squule . . . . . . . 417

Borläufer bes \$rotlus, Blutardus, Theodorus von

Afiue, Syrianus . . . . . . . . . . 418

Protlus . . . . . . . . . . 421

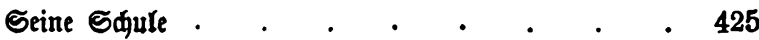

Damascius . . . . . . . . . 426

Simplicius . . . . . . . . 427

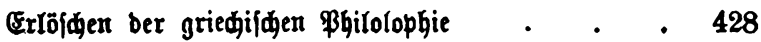

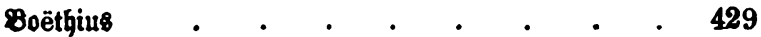

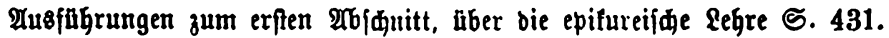

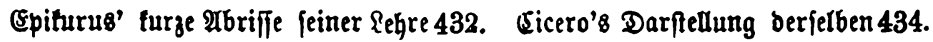

\&utretius' Darftellung 439 unb feine 2 uffaffung ber Sehre 492.

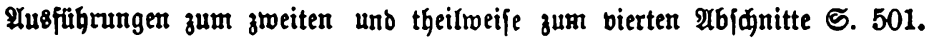
Zeno 501. Seine nädften Nadforger 510. (Shryfipps \&ehre von ben Affetten 511 u. 521; von ber Borftellung uno igren $\mathfrak{A r t e n} 513$. 3eno's, ธ̧һryfippus' und Diogenes' Sehren von bem Size ber Bernunft 
unb von ben affetten 516. Pofibonius' Rritil berjelben 528. Brud, füđe ber \$gyfit des \$ofidonius 535. Bofibonius' aftronomie und (selehrjamleit 540.

Die römijđen Stoiler. Senela S.541.

Senela's größere $\mathscr{A b g h a n d l u n g e n ~} 5.560$. Seine Briefe und ihre \&egre 566. Seine Eltettil 582. Die હdranken feiner Etbil 503. Seine Sdilderumgen und verlorenen Berfe 596.

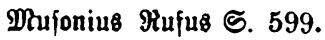

Epiftetus; jeine Blloung und bie (5)undlegung feiner Etthit S. 600. Sonderung bejien was im Bereide unfrer Selbftbefimmung liege und Deijen was nidt 606 . Die unferer Freibeit anbeimgeffellte Beberridung unferer Borftellungen und bie ridtige Entroidelung unfrer angeboretten Begriffe 607. Belebung unb Reinigung unfrer Bermunft 612. Ents widelung unb Siderung unfres freien 2 Bablbermögens 614 . Bereid unb Sqranten unferer freibeit 616. Ergebung in bie Fügungen ber göttliden Rorfegung 618. Epiftet's Gotteslegre unb frōmmigleit 620. Sein eigentgümlider Stanopuntt 623. Die Uebertieferungen feiner Segre 628.

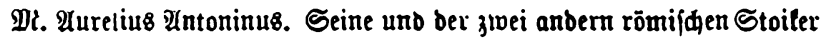
Darftellungsmeife 629. Die herallitijdje Brundegung feiner Sebre 630. Das über ben, etwigen ffuß̧ ber Dinge hinausreidende leben ber Ber. nunft 632 . Sonderung be\& Rörper\& und bes Şaudartigen von ber Ber, nunjt, und bie bödpe göttlide Bernunit 634 . Der unbebingt noth. wenbige Raulalnerus in ber 2 elt unb bas 2 alten ber göttliden Bor. fegung über bemjelben. Mealität ber 28 elt ber Dinge. Şarmonie und Einklany ber 93ermunft mit ber SRatur 635. Der wahre Bsotte8bienf 640. Der Tod und bas Edjiđfal ber Seele nad bem Tobe 641. Das (Eine was Roth thut 642 . Die liebe zur menfdliden Bemeinfdaft 645. Dic menfdlide Bemeinfiaft und ber Staat 647. Antoninus' fromme Ergebung; fein Begriff von ber Bottheit 649 . Seine Sdeu vor theoretifden Unterfudungeu, aud über unjere fortentwidelung

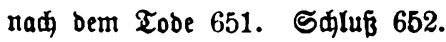

